INNOVATIONS IN PRIMARY CARE

\title{
Improved Outcomes Associated With Interprofessional Collaborative Practice
}

\author{
Thomas P. Guck, $P b D^{1}$ \\ Meghan R. Potthoff, PbD, \\ $A P R N^{2}$ \\ Ryan W. Walters, $P b D^{3}$ \\ Ann Fam Med 2019;17:S82. https://doi.org/10.1370/afm.2428.
}

\section{THE INNOVATION}

A private midwestern university and its academic health partner designed and implemented an interprofessional collaborative practice (IPCP) model within a family medicine residency and faculty practice at a new ambulatory care center (ACC). The purpose of our study was to evaluate the effectiveness of the IPCP model by comparing patient outcomes and costs during the year before the ACC opened (2016) and during the first year of the ACC under the IPCP model (2017).

\section{WHO \& WHERE}

We identified a cohort of high-risk patients to follow longitudinally who met 1 of the following clinical criteria in 2016: 3 or more emergency department visits in the first or second half of the year; a hemoglobin $A_{1 c}$ value of $9 \%$ or greater; or a LACE score (length of stay, acuity, comorbidities, emergency department visits) for readmission risk of 10 or greater. Patient demographics are provided in Table 1 (Supplemental Appendix at http://www.AnnFamMed.org/content/17/Suppl_1/S82/suppl/DC1). Patients were excluded if, by the end of 2017, they had switched to a primary care clinician outside the ACC, moved away, or died. From electronic health record reviews at the end of 2017, we determined that 276 patients met inclusion and exclusion criteria. The outcome measures included emergency department visits, hospitalizations, hemoglobin $A_{1 c}$ values, and patient charges.

\section{HOW}

Consensus definitions of interprofessional education and collaborative practice informed our IPCP model implemented

Conflicts of interest: authors report none.

\section{CORRESPONDING AUTHOR}

Thomas P. Guck, PhD

Creighton University School of Medicine

2412 Cuming St

Omaha, NE 68131

tpguck@creighton.edu at the ACC. ${ }^{1}$ We used a 3-pronged approach to building the model, including staff and clinician training, patient care preparation, and care conference planning. Implementation of the IPCP model intentionally established a culture that encouraged collaborative care. We provided 3 grant-supported training sessions centering around conflict engagement before and after the opening of the ACC. Daily huddles occurred for the entire team before the morning and afternoon clinic during which the team reviewed safety issues, recognized team members, highlighted care issues, and shared announcements. ${ }^{2}$ After the huddle and before the clinic, the care team undertook previsit planning that included a resident, a nurse practitioner, or a faculty member, who along with a medical assistant discussed patient needs, including any interprofessional care. IPCP professionals individually or in small combinations of professionals, known as teamlets, collaborated on patient care continuously thoughout the day. ${ }^{3}$ All ACC patients were introduced to IPCP team members with warm handoffs from the primary care clinician or medical assistant. Cases of patients from the high-risk registry were presented at weekly collaborative care conferences to the interprofessional team with recommendations for care placed in the electronic health record's collaborative care documentation.

\section{LEARNING}

In our high-risk cohort, IPCP implementation was associated with absolute reductions of $16.7 \%$ in emergency department visits, $17.7 \%$ in hospitalizations, $0.8 \%$ in hemoglobin $A_{1 c}$ levels, and $48.2 \%$ in total patient charges (Table 2 in the Supplemental Appendix at http://www.AnnFamMed.org/content/17/Suppl_1/ S82/suppl/DC1). Our results suggest an association between IPCP interventions and improved health outcomes and cost reductions ${ }^{4}$ that is consistent with findings from behaviorally integrated primary care ${ }^{5}$ and IPCP use in a family medicine residency training program. ${ }^{6}$ The observed benefits are also in keeping with the triple aim associated with US health care reform. Limitations include not addressing the funding of the IPCP model, ${ }^{7}$ lack of a randomized controlled design, and inability to examine the contributions of individual professions to outcomes. Despite these limitations, our results are encouraging, as we continue to follow this and a subsequent cohort to examine the intervention's sustainability and replicability.

References, affiliations, tables, key words, acknowledgments, and disclosures are presented in the Supplemental Appendix at http://www.AnnFamMed.org/content/17/Suppl_1/S82/suppI/DC1. 